\title{
Design of a Grating for Studying Smith-Purcell Radiation and Electron Acceleration
}

\author{
R.C. Fernow* \\ Brookhaven National Laboratory, Upton, NY 11973
}

\begin{abstract}
We describe work on the design of a diffraction grating which we intend to use for studying the production of Smith-Purcell radiation and the acceleration of electrons. We have developed computer codes based on the solution of the appropriate Maxwell's equations. A specific grating profile is given which is feasible to construct and which supports enhanced surface accelerating modes. We examine the possibility of using the Smith-Purcell effect to make a beam position monitor.
\end{abstract}

\section{INTRODUCTION}

In 1953 Smith and Purcell ${ }^{1}$ discovered that an electron passing over and close to the surface of a metallic reflection grating caused the emission of visible radiation. Purcell speculated that the radiation was due to the oscillating image charge in the grating that travelled along below the electron. Given this a number of people realized that the inverse effect must also exist, namely it must be possible to bring radiation down upon the grating and use the fields near the surface to accelerate particles. In $1980 \mathrm{Palmer}^{2}$ proposed a suitable geometry for laser acceleration and gave parameters for use with a $\mathrm{CO}_{2}$ laser.

It is important to be able to accurately calculate these radiation effects in detailed studies of the laser acceleration process. We would like to know the answers to questions such as how efficiently incoming radiation couples to a given grating surface, how much acceleration could be expected, how much energy the electron loses to Smith-Purcell radiation while it is traversing the acceleration structure, and if sufficient radiation is emitted in the visible region, for example, to make an effective monitor of the electron's interaction in the structure.

- This research supported by the C.S. Dept. of Energy under contract DE-AC02-76CII00016. 
The oscillating image charge model has the virtue that it is simple to use in calculations, but it is known to be incorrect since, for instance, it docs not predict the exponential fall off of the fields above the grating surface and it disagrees badly with measurements. A more sophisticated model by Smith and Purcell ${ }^{3}$ took into account the phase differences of separated surface elements when calculating the total field. This "effective charge model" does give the exponential fall off with distance above the surface, but it also disagrees with experimental measurements of angular distributions ${ }^{4}$.

Another class of models treats the problem from the point of viewof grating diffraction theory. In particular van den $\mathrm{Berg}^{5}$ has given a rigorous solution of Maxwell's equations for the problem of a point charge in constant rectilinear motion at a fixed distance above a perfectly conducting grating with arbitrary profile. In these models the traveling electron is considered to be the source of a spectrum of evanescent plane waves. These plane waves are incident upon the grating surface and under certain conditions can give rise to propagating diffraction modes. The outgoing diffracted plane waves constitute Smith-Purcell radiation. It should be emphasized that this radiation results from coupling with a longitudinal surface mode and hence it is distinct from other effects such as synchrotron radiation ${ }^{6}$.

In this paper we will be concerned with the design of resonant gratings which can be used to study Smith-Purcell radiation under similar conditions to those expected in a proposed laser acceleration experiment at the Brookhaven Accelerator Test Facility (ATF). In the next sections we give brief outlines of van den Berg's solution, the numerical procedures used to implement it, and the tests we used to verify that the code was working properly. We then describe some general characteristics of the radiation. We show that a suitable boundary surface can be found to produce a sharp Smith-Purcell peak perpendicular to the electron trajectory for $10.6 \mu \mathrm{m}$ radiation and that an accelerating surface mode is excited when plane wave radiation is brought onto the grating at this angle. We then describe a beam height monitor based on the detection of higher order visible Smith-Purcell radiation. Two computer codes have been developed to carry out these studies. One (PERCY) calculates the Smith-Purcell radiation emitted by an electron. The other (GERTY) calculates the evanescent surface modes excited by an incident plane wave. 


\section{VAN DEN BERG'S SOLUTION}

In Fig. 1 we show the coordinate systems used in this paper. An electron with velocity $v=\beta c$ travels in a vacuum parallel to the $\mathrm{z}$ axis and at a distance $\mathrm{y}_{0}$ above it. The perfectly conducting surface is periodic along $z$ with spatial periodicity $\mathrm{D}$. The structure is assumed to be uniform in the $x$ direction. The maximum height of the structure is $\mathrm{y}_{\max }$, so that the closest distance of the electron to the surface is $y_{0}-y_{\max }$. Fig. $1 \mathrm{~b}$ shows the definition of the angles $\eta$ and $\zeta$ which are used to specify the direction of outgoing radiation.

Van den Berg defines a current density $J$ from the moring point charge. The current density and the fields in space and time are given as a double Fourier transform of similar quantities in frequency, and wave number along the grooves of the grating ( $x$ direction). It follows from the Maxwell curl equations that all the components of $\mathrm{E}$ and $\mathrm{H}$ for the incident wave can be written in terms of $\mathrm{E}_{X}$ and $\mathrm{H}_{X}$. These two components satisfy separate and uncoupled Helmholtz equations in terms of $\mathrm{J}$. The solutions of these equations show that $\mathrm{E}_{X}$ and $\mathrm{H}_{X}$ are evanescent plane waves whose magnitude decreases exponentially in directions perpendicular to the electron trajectory.

The evanescent plane waves from the electron are then the incident radiation for a boundary value problem at the grating surface. The reflected fields are defined to be the total fields minus the incident fields. These fields are also Fourier transformed and have to satisfy source-free Maxwell curl equations. The tangential component of the total field has to vanish on the boundary of the perfectly conducting grating. All the field components can be expressed in terms of two unknown functions, $\Phi$ and $\Psi$. Because of the symmetry of the problem these quantities satisfy separate and uncoupled Helmholtz equations and boundary conditions. Thus the vector problem is reduced to two scalar ones, each corresponding to a different state of polarization.

The periodicity of the grating is taken into account by expanding the unknown functions in Floquet series along 2. A radiation Green's function is defined in terms of an infinite series of Floquet modes. A fixed number of these terms are explicitly calculated and the remainder estimated using asymptotic expansions. The Green's function is then used together with the boundary condition at the electron trajectory to derive the following integral erluations for $\Phi$ and $\Psi$ in terms of the integral along a single period of the boundary surface. 


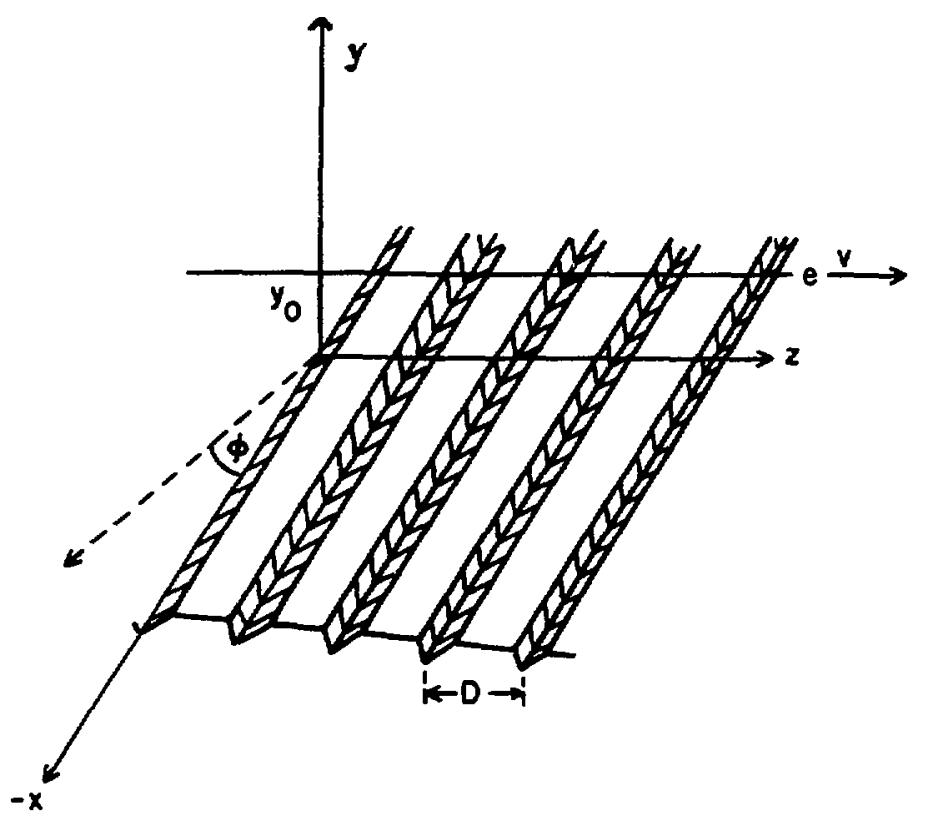

NORMAL

TO GRATING

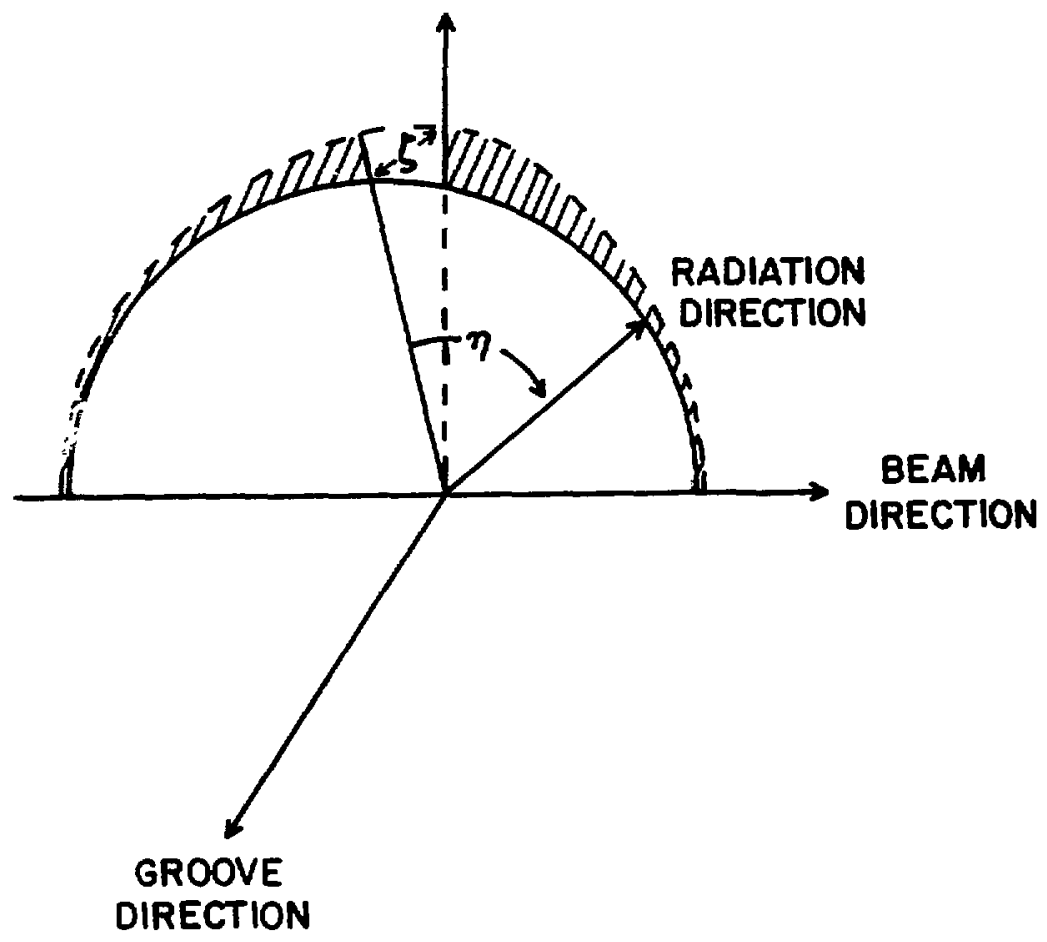

Fig. 1: Definition of coordinate systems used in this paper. 


$$
\begin{array}{rlrl}
\frac{1}{2} \hat{n}_{P} \cdot \nabla_{P} \Phi_{P}+P . V \cdot \int_{L}(\hat{n} \cdot \nabla \Phi)\left(-\hat{n}_{P} \cdot \nabla_{P} G\right) d s & =\hat{n}_{P} \cdot \nabla_{P} \Phi_{P}^{i} \\
& \frac{1}{2} \Psi_{P}+P \cdot V \cdot \int_{L} \Psi(\hat{n} \cdot \nabla G) d s & & =\Psi_{p}^{i}
\end{array}
$$

In these equations $s$ is the arclength, $L$ is one period along the grating boundary. $\mathrm{n}$ is the inward normal to the surface, the subscript $\mathrm{P}$ refers to a particular point on the surface, P.V. means the Cauchy principal value of the integral, $G$ is the Green's function, and $\Phi^{i}$ and $\Psi^{i}$ are known functions of the initial wavefunctions. Once the integral equations are solved for the two unknown functions, the field components of the modes can be calculated, and the radiated power can be found from the Poynting vector.

The heart of the numerical procedures used in PERCY and GERTY is the solution of the two complex integral equations of the second kind given in $\mathrm{Eq}$. (1). To solve these we break up the curve $\mathrm{L}$ into $\mathrm{N}$ equally spaced mesh points. In between adjacent pairs of mesh points we allow the unknown function to vary like a (linear or cubic) polynomial. Because of the periodicity there are $\mathrm{N}-1$ of these splines, the Nth mesh point being equivalent to the first one. We can convert the integral equation into a N-1 by N-1 system of linear algebraic equations by choosing the points $\mathrm{P}$ to correspond with the mesh points. The system of algebraic equations can then be solved by matrix inversion.

Both GERTY and PERCY have internal consistency checks based on the conservation of energy. For each polarization there is a relation between the $\mathrm{m}=0$ Floquet mode (energy into system) and the sum of the propagating outgoing Floquet modes (energy out of system). GERTY results for plane waves incident on sinusoidal and echelette gratings were compared with a number of results given in a paper by van den $\mathrm{Berg}^{7}$. The calculations of the relative power into the $\mathrm{m}=0,1$, and 2 Floquet modes for both the $\mathrm{E}$ and $\mathrm{H}$ polarizations of the incident radiation were found to be in good agreement. PERCY results for the production of Smith-Purcell radiation from a sinusoidal grating were compared with those of van den Berg ${ }^{5}$ for a number of cases. In general PERCY was able to reproduce van den Berg's results quite well ${ }^{4}$.

Of course the best test of any model is comparison of the calculation with experiment. The most detailed measurements of tle angular distribution of Smith-Purcell radiation appear to be that of Gover et al. ${ }^{8}$. Fig. 2 compares their measurernents of the angular distribution of power emitted into visible 


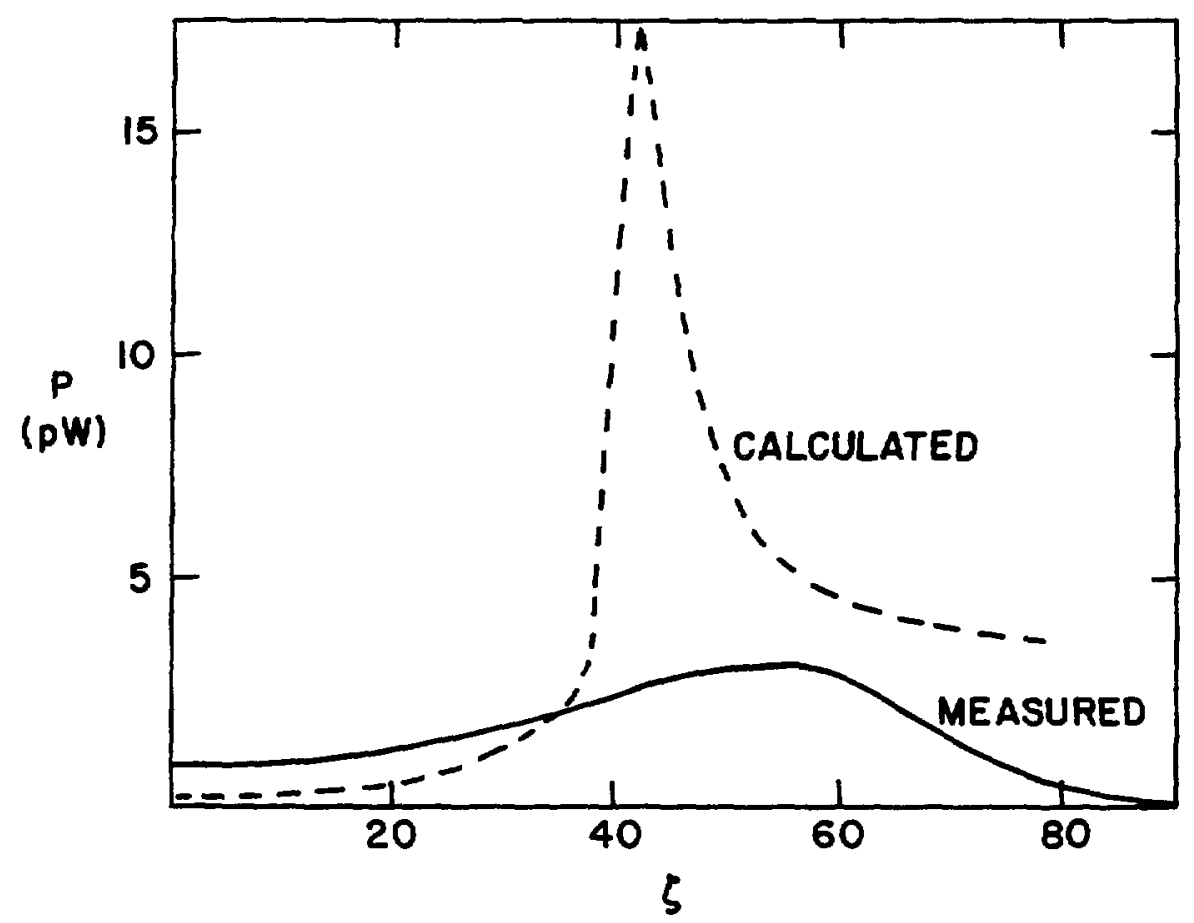

Fig. 2: Comparison of the angular distribution of measured Smith-Purcell power in the experiment of Gover et al. ${ }^{8}$ with calculations using PERCY. The measurements are for $\eta=-16^{\circ}$.

radiation with PERCY calculations for a uniformly distributed electron beam traveling over a sinusoidal grating. The angle $\zeta$ was defined in Fig. 1b. The calculation gives the correct qualitative behavior of the angular distribution. The absolute magnitude of the power agrees within a factor of 6 . This actually represents excellent agreement since (1) the perfect conductivity assumption is known ${ }^{9}$ to break down for wavelengths shorter than $4 \mu \mathrm{m},(2)$ the grating used by Gover et al. did not have a perfectly sinusoidal profile, and (3) convoluting the calculated spectrum with the finite solid angle acceptance of the detector would bring down the magnitude of the predicted peak signal.

\section{GENERAL PROPERTIES OF THE RADIATION}

Two properties of Smith-Purcell radiation are quite general and must be satisfied by any model which attempts to explain the effect: Smith-Purcell radiation satisfies a simple spectral relation and it exhibits a characteristic exponential fall off in intensity away from the grating surface. 


\section{Spectral relation}

The wavelength $\lambda$ of the emitted Smith-Purcell radiation must satisfy the equation

$$
\lambda=\frac{D}{-m}\left(\frac{1}{\beta}-\sin \eta\right)
$$

where $\mathrm{m}$ is the order (Floquet mode) of the radiation and the angle $\eta$ is defined in Fig. 1b. Smith-Purcell radiation can only occur when $\mathrm{m}$ is a negative integer. Eq. (2) shows that radiation at a fixed angle with respect to the beam direction has the same wavelength, regardless of the azimuthal angle around the beam. Thus looking back along the beam direction one would see a rainbow type of spectrum, a fact that has been confirmed by a number of investigators.

The minimum emitted wavelength occurs along the beam direction and for relativistic velocities we have $\lambda_{\min }=\frac{D}{2 m \gamma^{2}}$. The maximum wavelength, $\lambda_{\text {max }}=2 D$, occurs backwards to the beam direction.

\section{Exponential fall off}

The energy $W$ lost by a single electron falls off exponentially with its height above the grating

$$
W \propto \exp \left\{-\frac{\left(y_{0}-y_{\max }\right)}{h_{I N T}}\right\}
$$

where $y_{0}$ is the vertical position of the electron shown in Fig. la and $y_{\max }$ is the maximum height of the grating surface. The characteristic height for the energy loss to fall off by $\frac{1}{e}$ from its value on the grating surface is given by ${ }^{8}$

$$
h_{I N T}=\frac{D\left(\frac{1}{\beta}-\sin \eta\right)}{4 \pi m\left[\beta^{-2}-1+\cos ^{2} \eta \sin ^{2} \zeta\right]^{\frac{1}{2}}}
$$

Essentially, it is only the electrons that are within a distance $h_{I N T}$ of the surface that contribute to the production of radiation. This effective interaction distance is seen to be proportional to the electron velocity, the grating periodicty, and the order and production angles of the emitted radiation. In general higher order modes will tend to be suppressed because of the $\mathrm{m}$ in the denominator. In the limiting case when we consider the emission of radiation in the plane formed by the electron velocity and the normal to the grating (the $y-z$ plane where $\zeta=0$ ), we obtain the simple relation $h_{J N T}=\frac{3 \gamma \lambda}{4 \pi}$. 


\section{GRATING DESIGN}

We now consider gratings with the general form shown in Fig. 3. This figure shows one period of the grating. Electrons in a beam would pass from left to right across the figure. The grating grooves are perpendicular to the page. There are two grooves within each period, which can in general have different depths and can have their centerlines displaced from $\frac{D}{4}$ and $\frac{3 D}{4}$. This general grating shape was specifically chosen for ease of fabrication. It can be made using silicon etching facilities available at $\mathrm{BNL}^{10}$. The angle $\theta$ is chosen to be $54.7^{\circ}$ because this corresponds to the angle between the $\langle 100\rangle$ and $\langle 111\rangle$ planes of silicon and the etching can be readily controlled along this interface.

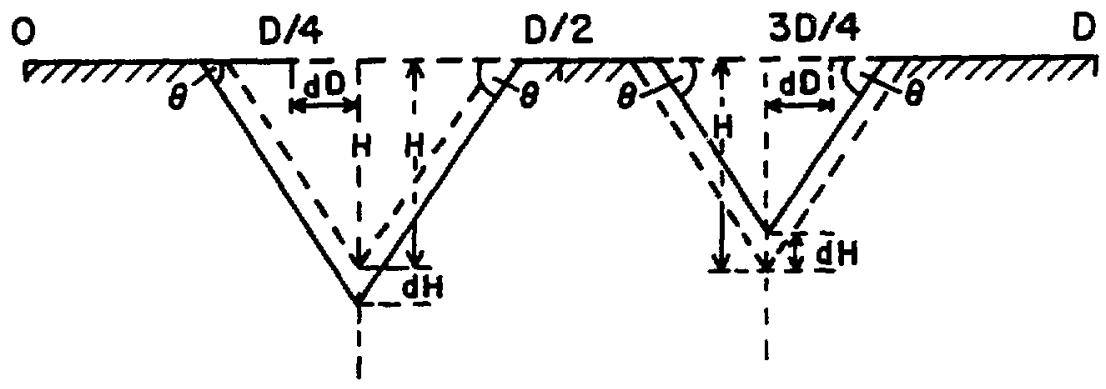

Fig. 3: General form of the grating shape studied in this paper. D: spatial periodicity, $\mathrm{H}$ : mean groove depth, $\mathrm{dH}$ : deviation from the mean groove depth, $\mathrm{dD}$ : deviation from symmetric groove spacing, $\theta$ groove angle.

For practical experimental reasons we are interested in coupling the radiation at right angles to the electron trajectory. For acceleration this could be achieved for example by using cylindrical optics to give a line focus of the incident radiation along the electron path ${ }^{2}$. We refer to the radiation in this case and in the corresponding case of Smith-Purcell radiation emitted perpendicularly to the electron direction as cylindrical radiation. From Eq. (2) we see that the period $\mathrm{D}=10.6 \mu \mathrm{m}$ is required for first order production of $10.6 \mu \mathrm{m}$ radiation at an angle of $90^{\circ}$ to the beam direction. (We note that we want a grating optimized for the production of $10.6 \mu \mathrm{m}$ radiation because we will use the same grating design for acceleration studies when the ATF $\mathrm{CO}_{2}$ laser is available. A large radiation peak implies strong beam-radiation coupling.) In order to couple to cylindrical radiation the depths of the two grooves must be slightly different ${ }^{2}$. Variations in the groove centerlines degraded the strength of the radiation so we set the parameter $\mathrm{dD}=0$. 


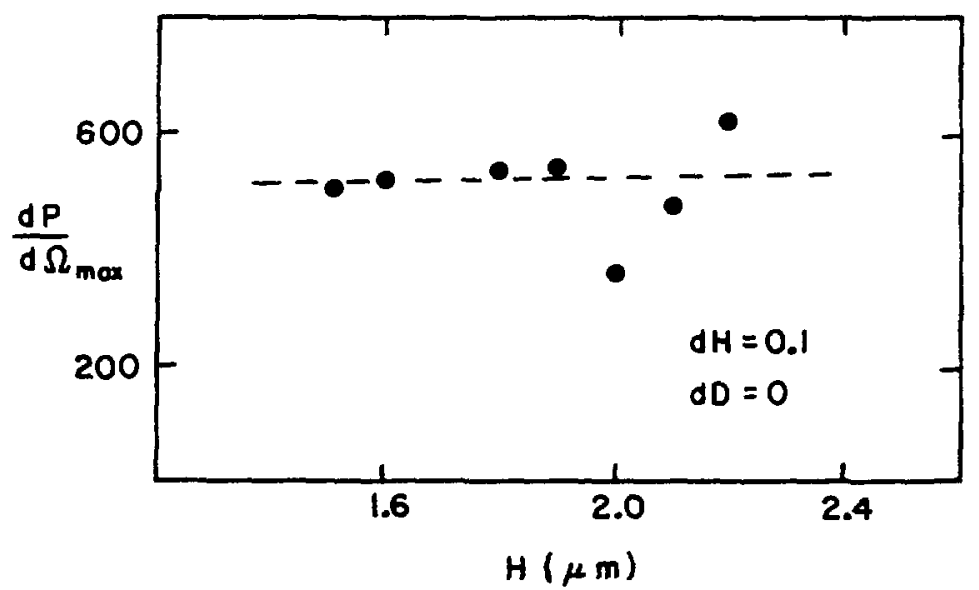

Fig. 4: Variation of the maximum emitted power (relative units) with the mean groove depth.

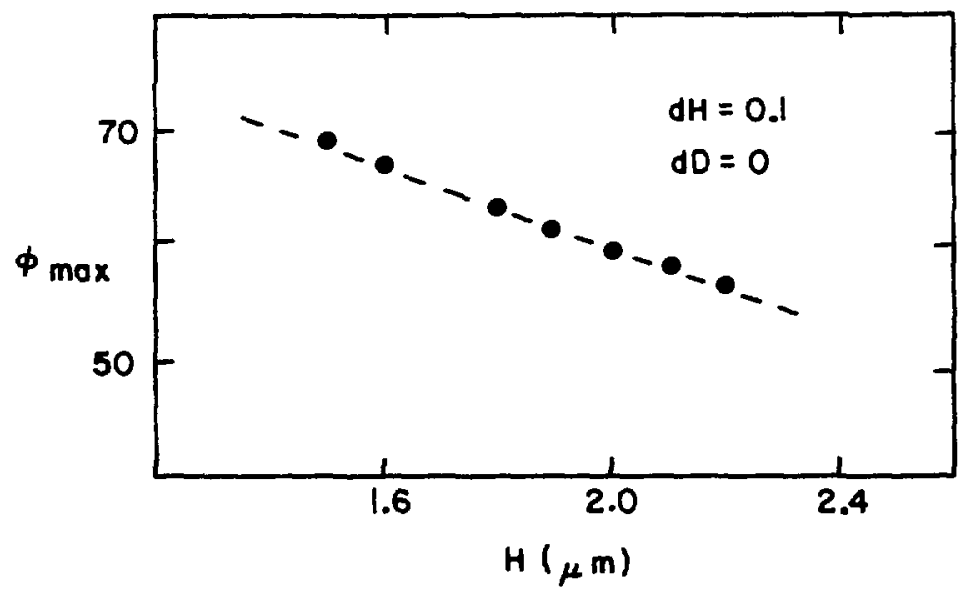

Fig. 5: The angle in degrees at which the maximum amount of power is emitted as a function of the mean groove depth.

Figs. 4-6 show how the power of cylindrical $10 \mu \mathrm{m}$ radiation varies with changes in the shape of the grating. The ordinates are proportional to the power from a beam of electrons with $\gamma=10$ uniformly distributed from the grating surface to infinity. However as we saw in Eq. (3), most of the power comes from those electrons that are within a few microns from the surface. Fig. 4 shows that the peak power is relatively insensitive to the mean groove depth $(\mathrm{H})$. Fig. 5 shows that the angle $\phi$ at which the maximum power is emitted varies linearly with $\mathrm{H}$. The angle $\phi$ is the angle up from the groove directiou in 
the plane perpendicular to the beam direction, as shown in Fig. 1a. Fig. 6 shows that the maximum power drops off rapidly when $\mathrm{dH}$ is increased. We also see that setting $\mathrm{dD}=0.2 \mu \mathrm{m}$ causes the maximum power to drop.

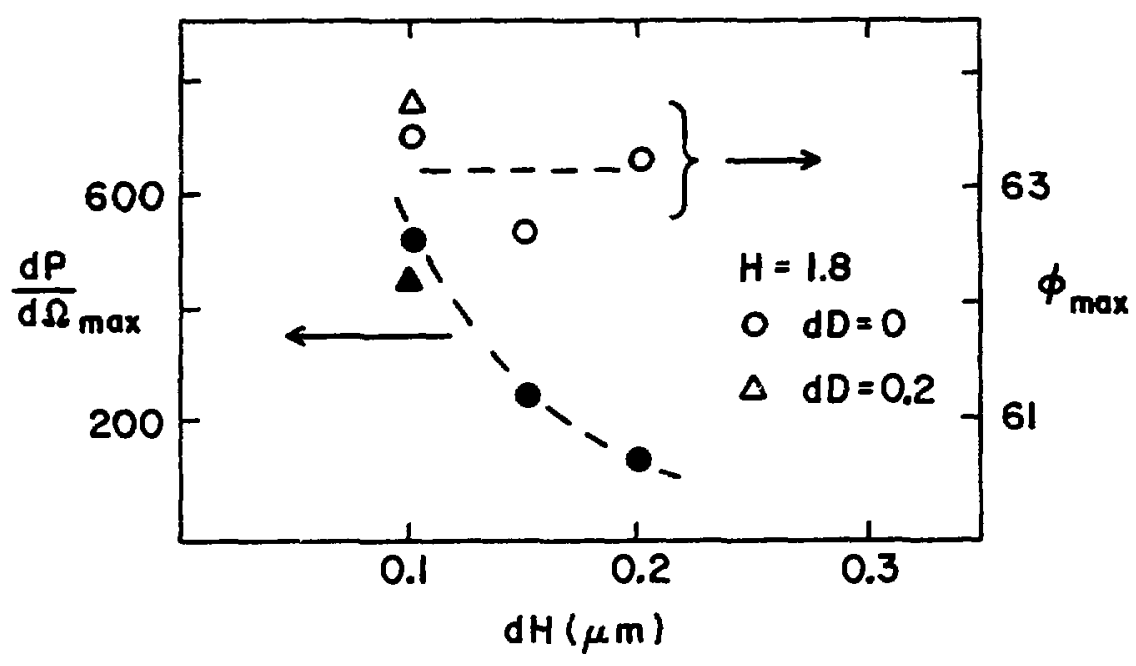

Fig. 6: Variation of the maximum emitted power (left scale) and the corresponding angle (right scale) as a function of the deviation from the mean groove depth. The triangles indicate the effect of breaking the symmetry in the groove spacing.

From a consideration of Figs. 3-6 we have chosen the parameter values given in Table 1.

Table 1: Grating parameters

\begin{tabular}{|l|l|}
\hline $\mathrm{D}$ & $10.6 \mu \mathrm{m}$ \\
$\mathrm{H}$ & $1.8 \mu \mathrm{m}$ \\
$\mathrm{dH}$ & $0.1 \mu \mathrm{m}$ \\
$\mathrm{dD}$ & 0 \\
$\theta$ & $54.7^{\circ}$ \\
\hline
\end{tabular}

\section{GRATING PERFORMANCE At $10.6 \mu \mathrm{m}$}

We consider here the coupling of the grating described in the previous section with $10.6 / \mathrm{m}$ radiation. We first examine the angular distribution of Snith-Purcell radiation produced by a bean of electrons. Then we consider 
the strength of the accelerating mode produced on the grating surface by an incoming plane wave at the appropriate angle.

\section{Cylindrical radiation}

Figure 7 shows the angular distribution of the radiated power at $10.6 \mu \mathrm{m}$ versus $\phi$ for a uniform beam. When $\mathrm{dH}=0.1$ the peak is quite sharp with a FWHM of only $0.14^{\circ}$. Increasing $\mathrm{dH}$ to $0.2 \mu \mathrm{m}$ causes the peak power to drop dramatically, causes the distribution to broaden, and causes the angle corresponding to the peak to shift. The radiated power is near 0 when $\mathrm{dH}=0$. The PERCY calculations shown in Fig. 7 should be accurate for the production of $10.6 \mu \mathrm{m}$ radiation since (1) the infinite conductivity assumption should be very good in this region of the infrared and (2) only one radiating mode is allowed.

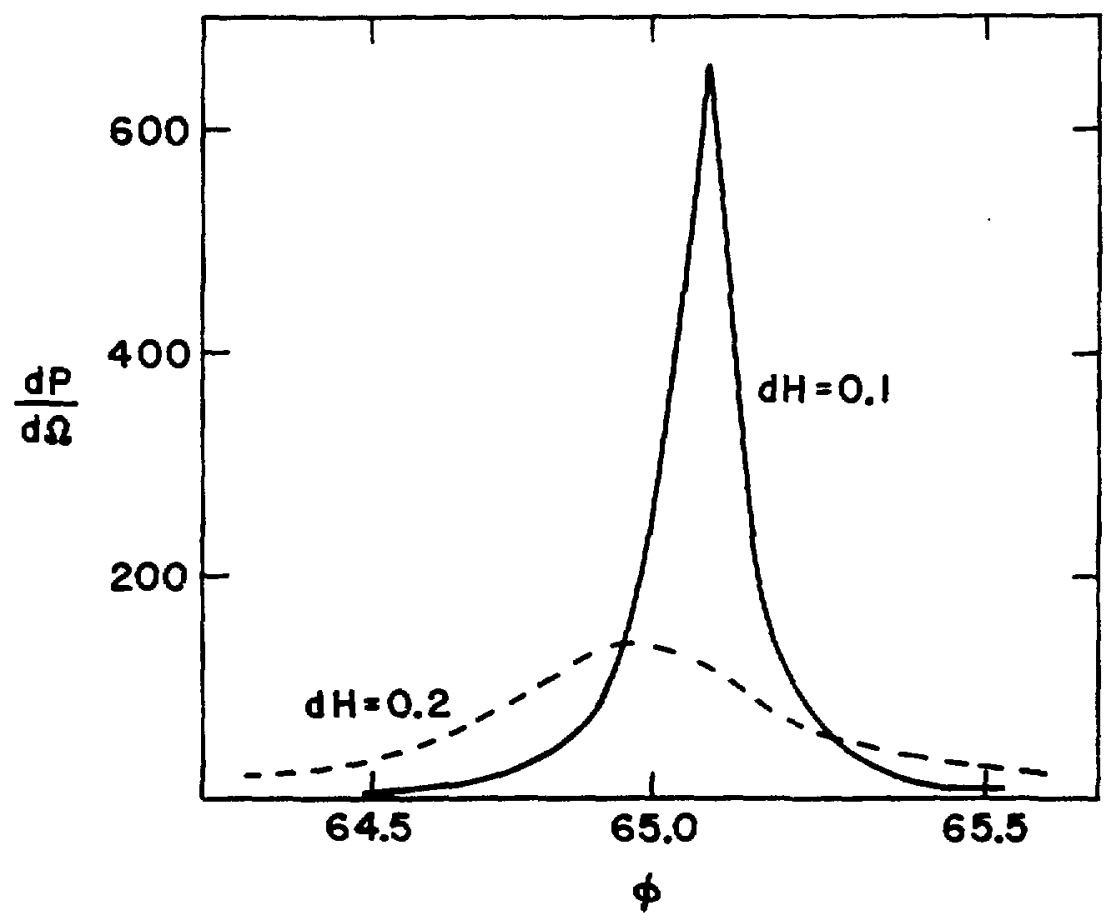

Fig. 7: The angular distribution of emitted Smith-Purcell power (relative units) for the grating defined in Table 1. 


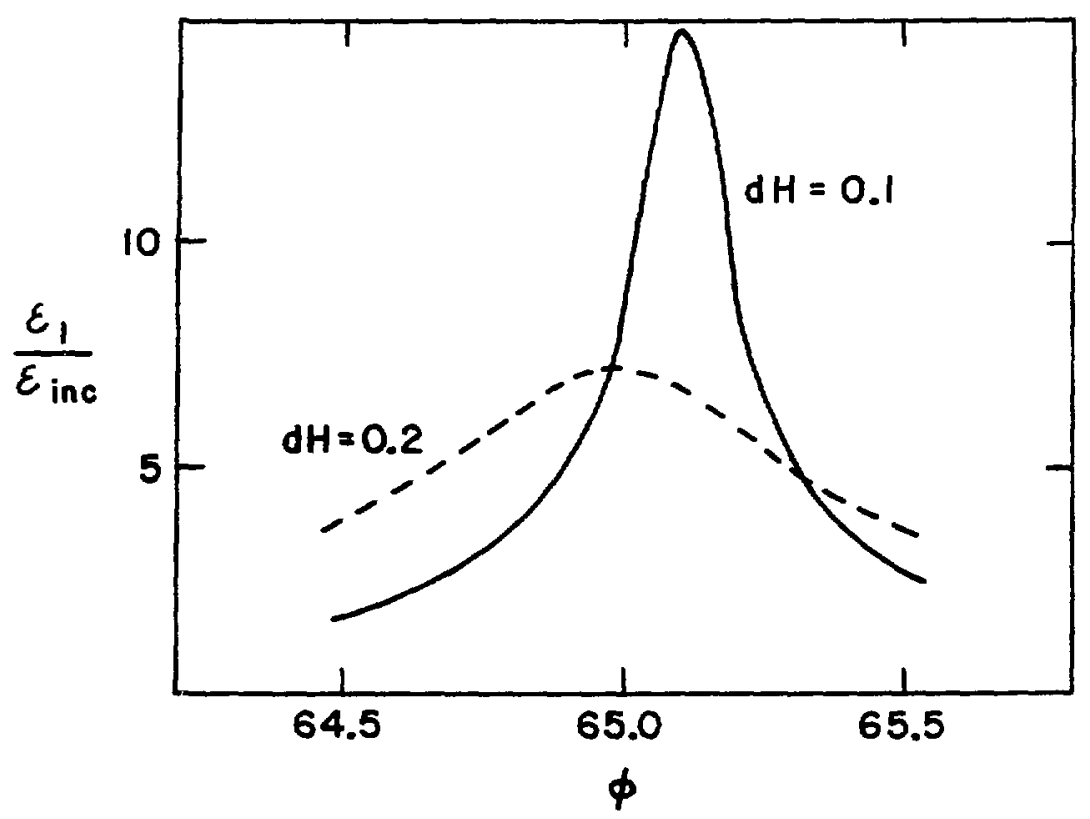

Fig. 8: The accelerating surface electric field enhancement as a function of the angle of incidence of an incoming plane ware.

\section{Accelerating surface modes}

By reciprocity if we bring a plane wave onto the grating at exactly the same angle as the peak in the radiation spectrum, we expect to create an accelerating surface mode that moves synchronously with the relativistic electron. For the configuration considered here there is only one propagating outgoing mode, namely the reflected radiation $(\mathrm{m}=0)$. The $\mathrm{m}=1$ mode is an evanescent surface wave which travels along with the electron with phase locity c. Pickup ${ }^{11}$ has shown that surface waves can satisfy this requirement provided the magnitude of the wavevector along the groove $\left(k_{X}\right)$ equals the magnitude of the wavevector perpendicular to the surface $\left(k_{Y}\right)$.

GERTY results for the ratio of the magnitudes of the accelerating electric field to the incident wave are shown as a function of the angle of incidence in Fig. 8. The incident radiation is linearly polarized with the electric field vector parallel to the electron trajectory. The calculations predict an accelerating field enhancement of about 15 for the grating described in Table 1. The actual enhancement will not be this large of course because of surface heating, reflection losses, and construction errors. Palmer ${ }^{2}$ has estimated that a more realistic culancement would be around 3 . The two curves in Fig. $S$ peal at the samc 


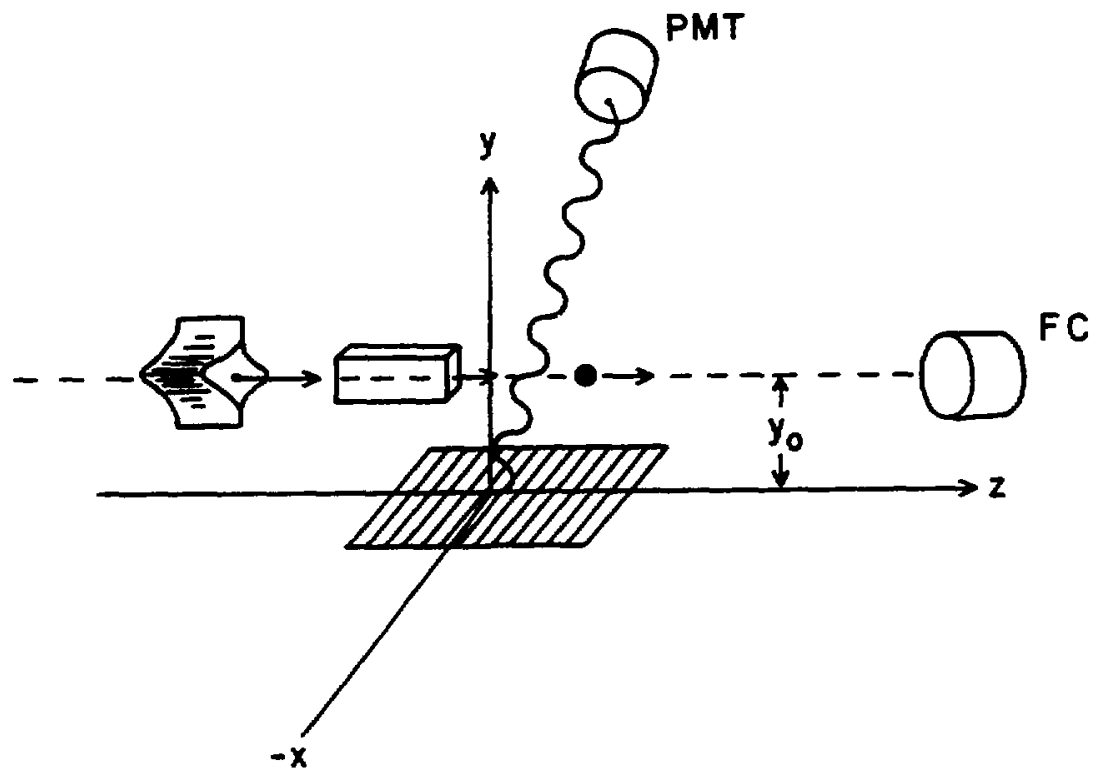

Fig. 9: A beam height monitor utilizing the Smith-Purcell effect. The figure shows a single electron, rectangular beam bunch, and a Gaussian beam bunch with a mean height $y_{0}$ above the surface. PMT: photomultiplier tube; FC: Faraday cup.

angles as the corresponding emitted powers in Fig. 7 . This demonstrates that we may search for efficient accelerating structures by looking for big emitted radiation peaks (reciprocity). The half width at half maximum of the $\mathrm{dH}=$ $0.1 \mu \mathrm{m}$ peak is $0.11^{\circ}$. The accuracy on the direction of the incident wave must be at least this good to adequately excite the accelerating mode.

\section{BEAM HEIGHT MONITOR}

One possible use of Smith-Purcell radiation is to provide a monitor for beam positioning. We saw in Eq. (3) that the energy loss by a passing charged particle changes exponentially with the distance to the grating surface. For the purpose of monitoring we are interested in the amount of radiation emitted in visible wavelengths because photomultiplier tubes (PMT) provide convenient, high gain detectors. Unfortunately the PERCY calculations become less reliable for this case. This is because, as we mentioned previously, the infinite conductivity assumption breaks down here and because the energy loss is distributed among many allowed radiating modes $(D \gg \lambda)$.

A posssible monitor configuration is show in Fig. 9. The radiation from a particle or beam bunch is detected by a PUT. A spectral filter can be 
used to select a narrow band of wavelengths. The PMT signal is normalized by measuring the incident beam intensity. A Faraday cup conld be used for this in certain applications.

Fig. 1b shows the definition of the angles used to describe the radiation direction. Fig. 10 shows the emitted power at $400 \mathrm{~mm}$ for the two strongest modes of the grating described in Table 1 . We see strong pealis near the plane containing the electron beam and the normal to the grating surfacc. particularly for the $\mathrm{m}=-4$ mode near $\eta=59^{\circ}$ and the $\mathrm{m}=-25$ mode near $4^{\circ}$.

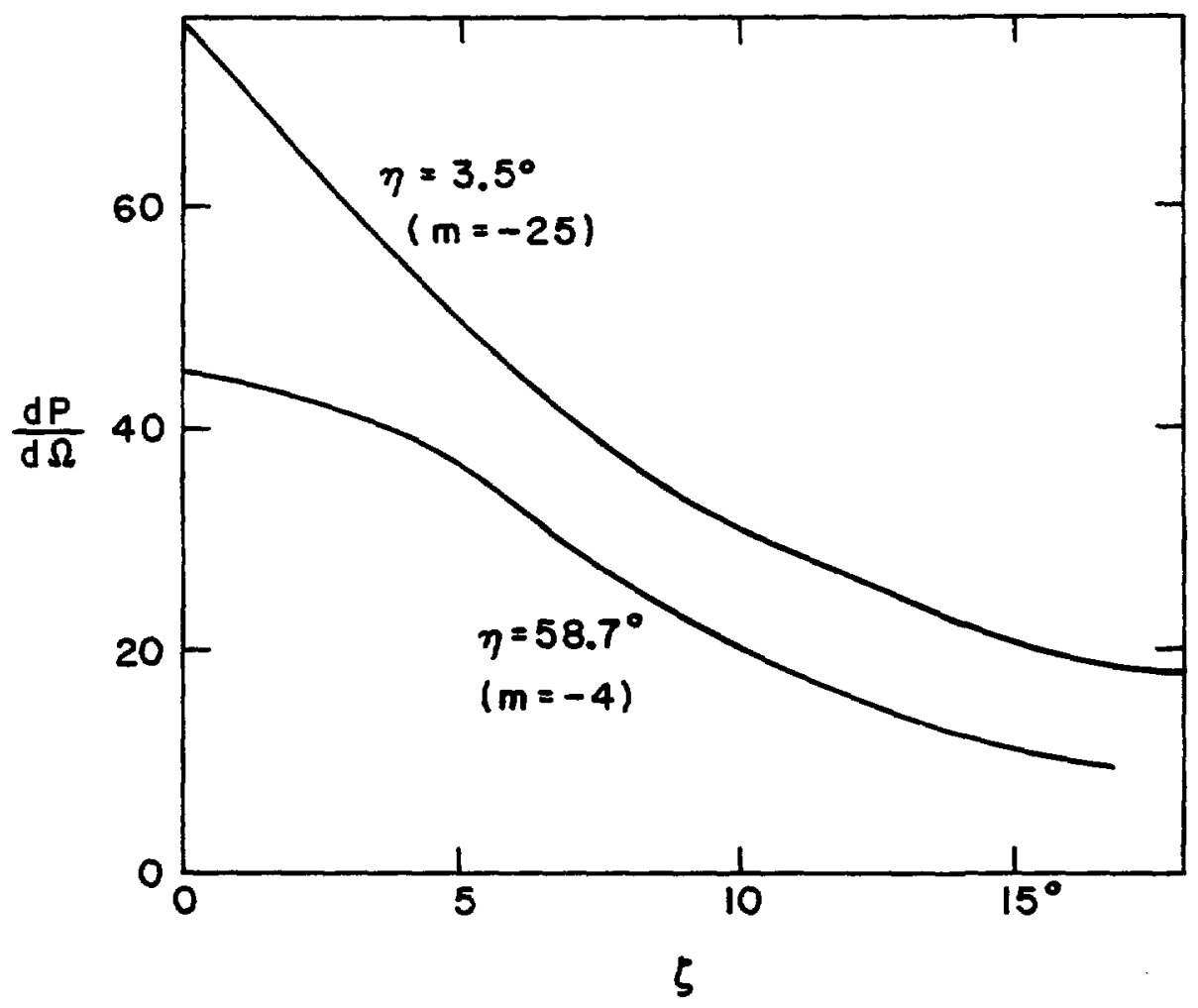

Fig. 10: Angular distributions of the two modes $(-m=4$ and 25) of the grating defined in Table 1 with the largest emitted powers (relative units) at 400 $\mathrm{nm}$.

We now consider a specific example of using the grating of Table 1 as a beam height monitor. The example parameters are given in Table 2.

The energy loss of a single electron into the solid angle $d \Omega$ can be written

$$
\frac{d W}{d \Omega}=F \exp \left\{-\frac{\left(y_{0}-y_{\max }\right)}{h_{\mathrm{INT}}}\right\}
$$


Table 2: Monitor parameters

\begin{tabular}{|l|l|}
\hline $\mathrm{m}$ & -25 \\
$\eta$ & $3.5^{\circ}$ \\
$\zeta$ & $0^{\circ}$ \\
$\lambda$ & $400 \mathrm{~nm}$ \\
$\mathrm{~L}$ & $10 \mathrm{~mm}$ \\
$\Delta \Omega$ & $15 \mathrm{msr}$ \\
$\gamma$ & 100 \\
$\mathrm{~h}_{\mathrm{INT}}$ & $0.32 \mu \mathrm{m}$ \\
$\left|\mathcal{R}_{n}\right|^{2}$ & 0.28 \\
$\mathrm{~N}_{0}$ & $150 \mu \mathrm{m}-\mu \mathrm{rad}$ \\
$\mathrm{N}_{0}$ & $10^{5}$ \\
\hline
\end{tabular}

where the quantity $F$ is defined as

$$
F=\frac{e^{2} m^{2}}{2 D \varepsilon_{0}} \frac{L}{D}\left|\mathcal{R}_{m}\right|^{2} \frac{\cos ^{2} \eta \cos ^{2} \zeta}{\left[\frac{1}{3}-\sin \eta\right]^{3}}
$$

The new quantities introduced here are the permittivity of free space $\varepsilon_{0}$, the total length of the grating $L$, and a radiation factor $\mathcal{R}_{m}$ which depends on the specific boundary surface ${ }^{12}$. The calculated energy loss for an electron travelling parallel to and $0.5 \mu \mathrm{m}$ above the surface for the conditions of Table 2 is $0.6 \mathrm{eV}$. Since this average loss is small compared to the energy of a $400 \mathrm{~nm}$ photon, this would not in general be a practical monitor.

The energy loss for a beam of particles uniformly distributed in height from the grating surface to infinity is

$$
\frac{d W}{d \Omega}=F \rho w h_{I N T}
$$

where $\rho$ is the number of beam particles per unit cross sectional area, and $w$ is the beam width (along the $\mathrm{x}$ direction) ${ }^{13}$.

Next consider beam bunches with a finite cross section. For a bunch uniformly distributed across a rectangular cross section (see Fig. 9) the energy loss can be written

$$
\frac{d W}{d \Omega}=F 2 N_{0} \frac{h_{I N T}}{s} \exp \left\{-\frac{\left(y_{0}-y_{\max }\right)}{h_{I N T}}\right\} \sinh \left(\frac{s}{2 h_{I N T}}\right)
$$

where $N_{0}$ is the total number of particles in the bunch and $s$ is the vertical extent of the bunch. Note that we have the relation $\lambda_{01}=\rho \mathrm{sw}$. For the conditions of 
Table 2 we take $\mathrm{s}=2 \sigma_{x}=5 \mu \mathrm{m}$. The energy loss can be written $\mathrm{W}[\mathrm{ev}]=$ $\neq \times 10^{i} \exp \left(-3.2 y_{0}[\mu \mathrm{m}]\right)$. This produces a measurable signal for bunch center heights from $2.5 \mu \mathrm{m}$ (the beam edge just touching the grating surface) to $5 \mu \mathrm{m}$.

Finally for a beam bunch with a bi-Gaussian distribution in the $x-y$ plane the energy loss is given by

$$
\begin{aligned}
\frac{d W}{d \Omega}= & F \frac{N_{0}}{2} \exp \left\{-\frac{\left(y_{0}-y_{\max }\right)}{h_{I N T}}\right\} \exp \left\{\frac{\sigma^{2}}{2 h_{I N T}^{2}}\right\} \\
& {\left[\operatorname{erf}\left(\frac{\sigma}{\sqrt{2} h_{I N T}}+\frac{1}{\sqrt{2}}\right)-\operatorname{erf}\left(\frac{\sigma}{\sqrt{2} h_{I N T}}-\frac{1}{\sqrt{2}}\right)\right] }
\end{aligned}
$$

where $\sigma$ is the standard deviation of the distribution in both $\mathrm{x}$ and $\mathrm{y}$, and we have assumed that the $N_{0}$ particles are contained within $\pm 1 \sigma$ contours. The coefficient multiplying the exponential in this case is about $1 / 2$ that for the square bunch. These results indicate that it may be possible to construct a monitor to accurately measure the beam centroid for low-emittance beams located within a half-wavelength of the grating surface.

\section{CONCLUSIONS}

We have shown that van den Berg's solution of the Smith-Purcell problem is a useful tool for understanding the coupling of a charged particle with radiation in the vicinity of an infinitely conducting periodic surface. We have shown specific surface profiles that will couple an electron to incident 10.6 um radiation. We have demonstrated the reciprocity between the angle of emission of Smith-Purcell radiation and the required angle for exciting a grating accelerator. The width of the angular distribution of the excited accelerating mode shows that the necessary accuracy in the incoming radiation angle is about $0.1^{\circ}$. The strength of the accelerating surface mode shows a significant enhancement over that of the incident radiation. Sufficient energy appears to be emitted in the visible to make a useful beam monitor.

\section{Acknowledgements}

I would like to thank J. Pasciak, H. Kirk, R. Palmer, J. Gallardo and $\mathrm{A}$. Gover for their assistance and/or useful suggestions.

\section{References}

1. S. Smith and E. Purcell, "Visible light from localized surface cliarges moving across a grating," Phys. Rev. 92, 1069 (1953). 
2. R.B. Palmer. "A laser driven grating accelerator." Part. Acc. 11. S1 (1980).

3. S. Smith. Visible light from localized surface charges moring across a grating, Thesis Harvard University, 1953.

4. R. Femow. "Effects of Smith-Purccll radiation on the laser acceleration experiment." CAP-ATF Technical Note 1, Brookhaven National Laboratory. 1987, unpublished.

5. P. van den Berg, "Smith-Purcell radiation from a point charge moving parallel to a reflection grating," J. Opt. Soc. Am. $\underline{63} 1588$ (1973).

6. A. Gover and P. Sprangle, "A unified theory of magnetic bremsstrahlung, electrostatic bremsstrahlung, Compton-Raman scattering, and CerenkovSmith-Purcell free electron lasers." IEEE QE-17 1196-1215. (1981).

7. P. van den Berg, "Diffraction theory of a reflection grating," Appl. Sci. Res. 242611071 .

\$. A. Gover. P. Dvorkis and U. Elisha. "Angular radiation pattern of SmithPurcell radiation.” J. Opt. Soc. An. B1 i23 (19S4).

0. R. Petit. Ed., Electromagnetic theory of gratings. R. Petit. Ed. (SpringerVerlag, 1980)p. 169.

10. The gratings are fabricated by John Warren of the BNL Instrumentation Division.

11. M. Pickup. "A grating linac at micorwave frequencies" in Laser Acceleration of Particles, C. Joshi and T. Katsouleas, Eds. (AIP Conf. Proc. No. 130 , 1985) p. 281.

12. We note that our derivation of the expression given by Eq. 6 differs from that of Gover et al. in reference $S$ by the factor $\mathrm{m}^{2} / 2$.

13. It is this quantity (without the constant and beam dependent factors) that is plotted in Figs. 4. 6, 7, and 10. 
2. R.B. Palmer. "A laser driven grating accelerator." Part. Acc. 11. $\$ 1$ (1980).

3. S. Smith, Visible light from localized surface charges moving across a grating, Thesis Harvard University, 1953.

4. R. Fernow, "Effects of Smith-Purcell radiation on the laser acceleration experiment," CAP-ATF Technical Note 1. Brookhaven National Laboratory, 1987, unpublished.

5. P. van den Berg, "Smith-Purcell radiation from a point charge moring parallel to a reflection grating," J. Opt. Soc. Am. $\underline{63} 1588$ (1973).

6. A. Gover and P. Sprangle, "A unified theory of magnetic bremsstrahlung, electrostatic bremsstrahlıng, Compton-Raman scattering. and CerenkovSmith-Purcell free electron lasers," IEEE QE-17 1196-1215. (1981).

7. P. van den Berg, "Diffraction theory of a reflection grating." Appl. Sci. Res. $\underline{\underline{24}} 2611971$.

8. A. Gover, P. Dvorkis and U. Elisha. "Angular radiation pattern of SmithPurcell radiation," J. Opt. Soc. A1n. B1 723 (1984).

9. R. Petit, Ed., Electromagnetic theory of gratings. R. Petit. Ed. ' SpringerVerlag, 1980) p. 169.

10. The gratings are fabricated by John Warren of the BNL Instrumentation Division.

11. M. Pickup, "A grating linac at microwave frequencies"in Laser Acceleration of Particles, C. Joshi and T. Katsouleas, Eds. (AIP Conf. Proc. No. 130, 1985) p. 281.

12. We note that our derivation of the expression given by Eq. 6 differs from that of Gover et al. in reference 8 by the factor $\mathrm{m}^{2} / 2$.

13. It is this quantity (without the constant and beam dependent factors) that is plotted in Figs. 4, 6, 7 , and 10.

\section{DISCLAIMER}

This report was prepared as an account of work sponsored by an agency of the United States Government. Neither the United States Government nor any agency thereof, nor any of their employees, makes any warranty, express or implied, or assumes any legal liability or responsibility for the accuracy, completeness, or usefulness of any information, apparatus, product, or process disclosed, or represents that its use would not infringe privately owned rights. Reference herein to any specific commercial product, process, or service by trade name, trademark, manufacturer, or otherwise does not necessarily constitute or imply its endorsement, recommendation, or favoring by the United States Government or any agency thereof. The views and opinions of authors expressed herein do not necessarily state or reflect those of the United States Government or any agency thereof. 